\title{
Evidence that brown is not an elemental color
}

\author{
PAUL C. QUINN \\ University of Iowa, Iowa City, Iowa
}

\author{
and \\ J. L. ROSANO and B. R. WOOTEN \\ Brown University, Providence, Rhode Island
}

\begin{abstract}
Two experiments using the continuous judgmental color-naming technique (Sternheim \& Boynton, 1966) were performed to investigate possible response biases in an earlier investigation of the possible elemental nature of brown (Fuld, Werner, \& Wooten, 1983). We manipulated the order in which the response categories were presented by attempting to build in a maroon category dependence (Experiment 1) and by avoiding a brown category dependence (Experiment 2). The results indicate that the order in which the response categories become available can influence color-naming results; they also indicate that brown is not an elemental color. Yellow and black were reconfirmed as elemental colors, thus upholding the assumption of Hering opponent process models that there are six elemental colors.
\end{abstract}

A historically controversial question in the area of visual perception concerns the minimum number of color sensations that are considered to be elemental in that they cannot be broken down into more basic components. Under the assumption that some color sensations are not unique, but are, rather, combinations of other more basic sensations, the questions of interest have focused on just what the number and identity of the basic color sensations are. Many traditional models of color perception, as well as the more modern opponent-process models, have assumed that there are six elemental color sensations: black, white, blue, green, yellow, and red (see Boring, 1942; Hurvich, 1981). The results of a number of recent studies that employed Sternheim and Boynton's (1966) continuous judgmental color-naming technique have largely upheld this assumption (Fuld, Wooten, \& Whalen, 1981; Quinn, Wooten, \& Ludman, 1985).

According to the criterion of the continuous judgmental color-naming technique, a color sensation is considered to be elemental if its usual color term cannot be replaced by some combination of other color terms. In the initial application of this criterion, Sternheim and Boynton (1966) showed that orange was not an elemental color sensation; stimuli normally associated with the color term orange were described with some combination of the terms red and yellow. Similarly, stimuli associated with the color term purple can be described with a combination of the terms red and blue and stimuli labeled with

Preparation of this manuscript was supported by BRSG 507 RR 07035 20 from the Biomedical Research Support Group Program, Division of Research Resources, National Institutes of Health, and by a University of Iowa Old Gold Summer Fellowship awarded to Paul C. Quinn. A portion of this work was presented at the 1984 Optical Society of America meeting in San Diego, CA. P. C. Quinn's mailing address is Spence Laboratory of Psychology, Department of Psychology, University of Iowa, Iowa City, IA 52242. the color term gray can be described with a combination of the terms black and white, thus suggesting that purple and gray are nonelemental color sensations (Fuld et al., 1981; Quinn et al., 1985). The color sensations of red, yellow, blue, black, and white, however, are elemental; they cannot be described with some combination of other color terms. Although green has not been examined in this manner, most theorists assume that it, too, is elemental. Empirical work in this area tends to confirm the assertion of opponent-process theories in the tradition of $\mathrm{Au}$ bert and Hering (see Boring, 1942) that there are six elemental colors.

A more puzzling issue has been the nature of the color brown. At one level, brown may easily be described as a region in color space "between red and yellow in hue, of medium to low lightness and of moderate to low saturation'" (Webster's Third New International Dictionary of the English Language, 1961). Using psychophysical procedures, Bartleson (1976) verified this common dictionary definition. A more fundamental question is whether brown is elemental or a perceptual composite of two or more elemental colors. Although Hering $(1920 / 1964)$ recognized that "the distinguishing feature of brown, namely, its blackness, never shows up clearly as an independent quality added to the yellow hue" (p. 58), he clearly viewed brown as a yellow-black or a yellow-red-black. This also seems to be the view of the Committee on Colorimetry of the Optical Society of America (1963), which defines it as the "color name, for perception of dark yellow or orange." On the other hand, some have suggested that brown may constitute an elemental color sensation (Padgham \& Saunders, 1975; Ratliff, 1976). Fuld et al. (1983) employed the continuous judgmental color-naming technique to empirically evaluate the nature of brown. Using a 580-nm test light surrounded by a variable-intensity white annular field, 
Fuld et al. found that when the surround field was of an intermediate intensity, subjects required the term brown, in addition to the terms yellow and black, to completely describe the color of the test field. On the basis of this finding, Fuld et al. concluded that brown might be an elemental color. Such a finding is significant in that it calls into question a fundamental assumption of opponentprocess theory.

As an alternative explanation of their results, Fuld et al. (1983) considered the possibility that "subjects were unwilling to describe certain stimuli completely in terms of black and yellow because of the subjects' knowledge of using the alternative name, brown" (p. 636). This possibility arose because of the successive nature of the experimental sessions. In a preliminary session, subjects were asked to judge the percentage of brown present in various wavelengths surrounded by a fixed white field. This was necessary to find the wavelength that gave the best brown for each subject. In the next session, subjects were again allowed to use the term brown as well as the terms yellow and black to describe the stimuli. In subsequent sessions, the subjects were allowed to use only the terms black and yellow; the term brown was prohibited. By introducing brown as a response category in the first two sessions, a response bias may have been created on the part of the subjects. That is, in the later conditions, the subjects may have been influenced by the knowledge that if the term brown were available as it had been earlier, then it could be used to describe at least a percentage of the color of the test stimulus. As a result, test stimuli initially described with the term brown would not be fully describable with the terms black and yellow.

The purpose of the present study was to systematically investigate the possible response biases that make it difficult to interpret the Fuld et al. (1983) results unambiguously. If the continuous judgmental color-naming technique is sensitive to the order of availability of color names (at least under some conditions), then it should be possible to build in a response bias for a color sensation that has previously been shown to be nonelemental. In Experiment 1, the stimulus was a 650-nm test light enclosed by a variable-intensity surround annulus. Fuld et al. found that a similar stimulus (i.e., a $660-\mathrm{nm}$ test field) was completely describable with the terms red and black. In Experiment 1, we attempted to build in a "maroon" response dependency by allowing the stimuli to be described with the terms black, red, and maroon in the early sessions. We then removed maroon as a response category in a later session. In Experiment 2, brown stimuli were evaluated. In contrast to the Fuld et al. design, only the terms black and yellow were available to the subjects in the first session; the term brown was added in a later session. By not allowing the term brown to be used by the subjects until the later session, biases such as those that may have been induced by the Fuld et al. design should be eliminated. The results indicate that brown is not an elemental color sensation, reaffirming the notion that black, white, blue, green, yellow, and red are the only elemental color sensations.

\section{EXPERIMENT 1}

\section{Method}

Subjects. Three undergraduate students ( 2 males and 1 female) volunteered as subjects. The subjects had little or no knowledge of color science, and were naive with respect to the hypotheses being tested. All subjects had normal color vision as assessed by the Ishihara pseudoisochromatic plates.

Apparatus. Two channels of a four-channel Maxwellian-view optical system (described in Knoblauch, 1981) were used. Light in one channel emanated from a xenon lamp source and provided a central test field that was rendered monochromatic by a grating monochrometer. Light in the second channel emanated from a second xenon lamp source and provided a surround annulus that was rendered white by interposing Wratten color-balancing filters until it was judged by the experimenters to be devoid of hue. The centersurround configuration was achieved by using a Lummer-Brodhan cube. The central test field was oval in shape, with the major axis along the vertical subtending a visual angle of $2.36^{\circ}$; the horizontal minor axis subtended $1.44^{\circ}$. The outside diameter of the annulus was $5.58^{\circ}$ of visual angle, and its inner edge was contiguous with the oval test field. The exit pupil of the optical system was $1.5 \mathrm{~mm}$ in diameter and was centered in the subject's right pupil. An adjustable bite-bar and headrest assembly was used to align and stabilize the subject. Retinal illuminance was determined by Westheimer's (1966) method.

Procedure. To generate the red-maroon-black series, the wavelength $(650 \mathrm{~nm})$ and illuminance of the central test field were fixed and the illuminance of the white annulus was varied. The retinal illuminance of the surround varied from 1.5 to $3.6 \mathrm{log}$ Td. Since the surround levels were chosen for optimum perceptual spacing, they were not equally spaced physically. The 10 retinal illuminance values of the surround were (in $\log$ trolands) $1.50,1.90,2.30,2.50$, $2.70,2.90,3.10,3.30,3.50$, and 3.62. The brightness of the monochromatic test field was matched to the 2.5-log-Td surround by an experienced observer, using a heterochromatic matching technique.

The experiment consisted of three sessions, which differed in the names available to describe the central test field: Session 1, maroon; Session 2, maroon, red, yellow, and black; and Session 3, red, yellow, and black. Six blocks were presented in each session, and the 10 stimuli were presented in a random order within a block. Each stimulus was flashed for $1.5 \mathrm{sec}$ to the fovea of the subject's right eye. The interstimulus interval was $20 \mathrm{sec}$, unless the subject needed a second stimulus viewing, in which case the stimulus was repeated after approximately $10 \mathrm{sec}$. This occurred infrequently.

Prior to each session, the subjects were dark adapted for $10 \mathrm{~min}$. During this time they were given their instructions, which were identical for each session. They were asked to assign percentages to the available response categories that represented the amount of each color sensation perceived in a given test light. They were further told that these percentages did not have to sum to $100 \%$ if the names they were allowed to use in a session were insufficient to completely describe the stimulus.

\section{Results and Discussion}

It may be helpful at this point to restate the empirical criterion that would establish maroon as an elemental color. In each session, the subjects were given a number of color terms to describe the series of stimuli. If the avail- 
able color terms were insufficient to completely describe the stimuli, a computed function would be obtained that would represent the amount that the sum of the percentages assigned to the color terms for each stimulus fell short of 100. Maroon would be considered elemental if, for a session in which its name was prohibited from use (Session 3), a computed function could be obtained that matched the maroon response function when the color name was allowed (Sessions 1 and 2). Maroon would be considered nonelemental if any computed functions generated when the term was not allowed (Session 3) did not match with the response functions for maroon when the term was allowed (Sessions 1 and 2).

The results of Experiment 1 are shown in Figure 1, where percent color is plotted as a function of the retinal illuminance of the surround. ${ }^{1}$ The top panel of the figure displays the response functions obtained from Sessions 1 and 2 , in which the maroon response category was available alone or in combination with red, black, and yellow; the bottom panel contains the response functions obtained in Session 3, in which maroon was not allowed. Considering first the maroon response functions from Session 1, one can see that as the retinal illuminance of the surround was increased, the maroon response for 1 subject increased (G.A.G.), whereas the maroon responses for the other 2 subjects increased and then decreased. In Session 2, red, maroon (for Subject G.A.G.), and yellow were the predominant responses at low values of surround illumination; they were replaced by predominantly $m a$ roon and black responses at high values of surround illumination. The maroon response functions from Sessions 1 and 2 corresponded well for 2 of the 3 observers (G.A.G. and S.J.T.), but not for the third observer (T.B.D.).

The results from Session 3 are shown in the bottom panel of Figure 1. For all 3 observers, red and yellow responses were common at low values of surround illumination; these were replaced by a high proportion of black responses at high values of surround illumination. Although 2 of the observers showed negligible computed functions, the third observer (S.J.T.) displayed a computed function that corresponded well with the maroon response functions shown in the top panel. Thus, for 2 of the observers, the early availability of the term $m a-$

\section{Percent}
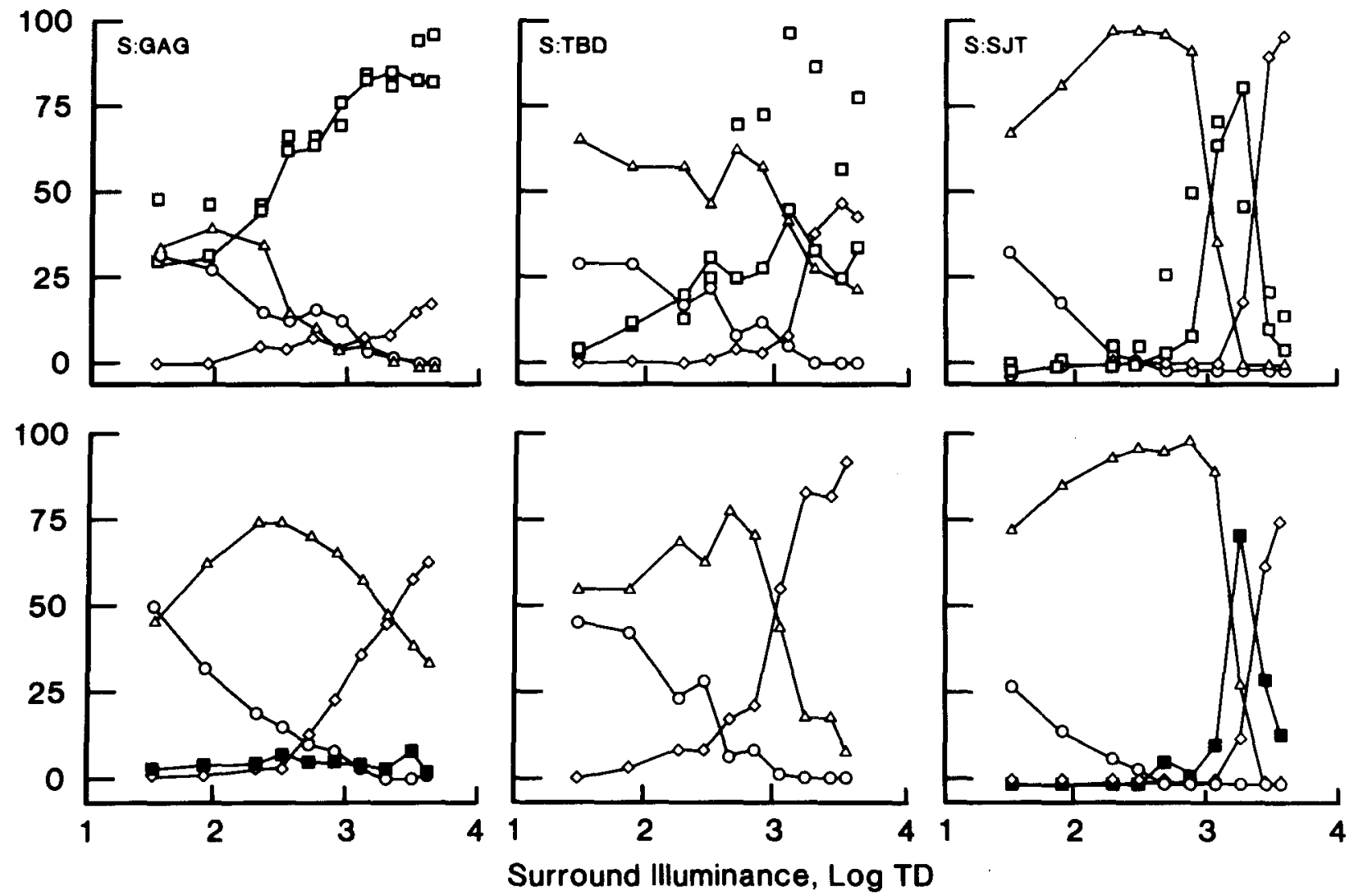

Figure 1. (Top panel) Color-naming responses from Sessions 1 and 2 of Experiment 1 for the 3 subjects (response categories: Session 1, maroon; Session 2, maroon, black, red, and yellow). Session 1 maroon responses are represented by the unconnected squares; Session 2 maroon, black, red, and yellow response functions are indicated by connected squares, diamonds, triangles, and circles, respectively. (Bottom panel) Color-naming responses from Session 3, with black, red, and yellow response functions indicated by unfilled diamonds, triangles, and circles, respectively. Computed functions are indicated by filled squares. 
roon did not result in a dependency on this term, because these observers could completely describe stimuli that had previously been described with the term maroon with the terms red, black, and yellow. For the third observer, however, the early availability of the term maroon did result in a response dependency on this term, as evidenced by the close correspondence between the response functions obtained when the term was available and the computed function obtained when the term was unavailable. After using the term in Sessions 1 and 2, Subject S.J.T. was not willing to completely describe the test fields as a combination of yellow, red, and black.

The results of Experiment 1 indicate that the results obtained with the continuous judgmental color-naming procedure can sometimes be affected by the order in which the color terms are made available to the subjects. Fuld et al. (1983) showed that a similar maroon series was completely describable with the terms red and black. After his initial experience using the term maroon to describe these lights, however, Subject S.J.T. was subsequently unwilling to completely describe them as red and black, with the result that his computed function matched his $m a-$ roon response function. A term previously shown to be redundant by Fuld et al. (since red and black were necessary and sufficient to describe the lights) had become necessary, misleadingly implicating maroon as an elemental color sensation. Such a result raises the possibility that at least some of the subjects in the later color-naming sessions of the Fuld et al. study were performing with an induced response dependency on the term brown. Experiment 2 addressed this possibility more directly.

\section{EXPERIMENT 2}

Experiment 2 was designed to reevaluate the possible elemental status of the color brown by attempting to replicate the results of Fuld et al. (1983) with the procedural modification that the term brown be unavailable to the subjects in the early color-naming sessions. The subjects' responses during these sessions would thus not be susceptible to the kind of bias introduced by prior availability of the term and demonstrated for Subject S.J.T. in Experiment 1. The design of Experiment 2 also allowed us to reconfirm the previously documented elemental nature of yellow (Sternheim \& Boynton, 1966) and black (Quinn et al., 1985).

\section{Method}

Subjects. Three female undergraduates served as subjects. As was true for the subjects in Experiment 1, they were naive with respect to the hypotheses being tested and had normal color vision, as assessed by Ishihara pseudoisochromatic plates.

Apparatus. The apparatus was identical to that employed in Experiment 1.

Procedure. To generate the yellow-brown-black series, the wavelength of the central test field was fixed at $570 \mathrm{~nm}$ and, as in Experiment 1, the illuminance of the surrounding annulus was varied. Eleven test lights that could be distinguished on the basis of the value of their surround illumination were used. They included the 10 stimuli with the same values of surround illumination used in Experiment 1 and 1 additional stimulus that allowed the subjects to view a broad perceptual continuum ranging from yellow to black. The 11th stimulus was added at the black end of the perceptual continuum and was created by fixing the value of surround illumination at $3.62 \log \mathrm{Td}$ and by blocking light in the central test field. In Figures 2 through 6, this stimulus is distinguished from the other stimuli by the placement of breaks in the appropriate places on the response functions and along the $x$-axis.

Experiment 2 consisted of five sessions, each run on a separate day. Each session was conducted in the same manner as in Experiment 1 . Six responses were obtained for each of the test stimuli, and the same blocking and randomization procedures were used to present the stimuli. The sessions varied in terms of the allowed response categories: Session 1, black and yellow; Session 2, yellow; Session 3, black; Session 4, black, yellow, and brown; Session 5, black and yellow. The color term red was also available in all sessions, but was used infrequently by the subjects. The same instructions given in Experiment 1 were given in Experiment 2: The subjects were told to assign percentages to the available response categories to describe the amount of the corresponding color sensations that they perceived in a given stimulus; they were also told that the percentages did not have to add up to $100 \%$ if the allowed color terms were insufficient to describe a stimulus. Throughout the sessions, the subjects were reminded to describe only the appearance of the central test field, that is, to ignore the surround.

\section{Results}

The elemental versus nonelemental status of the black, yellow, and brown color sensations can be determined by comparing response functions for these sensations with computed functions obtained when the terms were unavailable. The previously demonstrated elemental nature of black can be reconfirmed by comparing the black response functions obtained in Sessions 1 and 3 with the computed functions obtained in Session 2, in which the term was not allowed. Similarly, the previously documented elemental nature of yellow can be reconfirmed by comparing the yellow response functions in Sessions 1 and 2 with the computed functions obtained in Session 3, in which the term was not allowed. Most importantly, the possible elemental nature of brown can be evaluated by comparing the brown response functions obtained in Session 4 with the computed functions obtained in Session 1, in which only the terms black, yellow, and red were allowed.

The results from Session 1, in which the terms black, yellow, and red were available, are shown in the top panel of Figure 2, where percent color is plotted as a function of surround illuminance. For all 3 subjects, as the illuminance of the surround was increased, reliance on the yellow response category was replaced by reliance on the black response category. Only 1 subject (F.F.) used the red response category, and she did not use it extensively. The bottom panel of Figure 2 contains the crucial comparison between the computed functions obtained in Session 1 and the brown response functions obtained in Session 4. For all 3 subjects, there is a complete lack of correspondence between the computed and brown response functions. The computed functions from Session 1 were either essentially negligible (Subjects B.L. 
and F.F.) or unobtainable (Subject S.B.), in contrast to the orderly brown response functions that peaked for all 3 subjects at an intermediate value of surround illumination. The lack of agreement between the computed and the brown response functions provides strong support for the claim that brown is not an elemental color.

The top panel of Figure 3 displays the yellow response functions obtained in Session 2, in which the terms yellow and red were allowable. All three functions reflect a decrease in usage of the yellow response category as surround illumination was increased. Only Subject F.F. used the red response category. The bottom panel of Figure 3 contains the computed functions obtained in Session 2 and the black response functions from Sessions 1 and 3. The three functions for each subject match quite well. The excellent agreement between the black response functions and the computed functions obtained when the black category was not available provides a second empirical demonstration that black is an elemental color sensation.

The black response functions from Session 3 (allowed names: black and red) are displayed in the top panel of Figure 4. These functions appear as mirror images of the yellow response functions obtained in the previous ses- sion. They reflect a gradual increase in usage of the black response category with an increase in surround illumination. The bottom panel of Figure 3 contains plots of the computed functions obtained in Session 3 and replots of the yellow response functions obtained in Sessions 1 and 2 . It is quite clear that the three functions for each observer correspond closely. This agreement between the yellow response functions and the comptued functions obtained when yellow was unavailable reaffirm the contention that yellow is an elemental color.

The brown response category became available (along with the black, red, and yellow categories) in Session 4; the results from this session are shown in Figure 5. It can be seen that the subjects used the term brown, especially at intermediate surround illuminances, to describe lights previously described completely with the terms yellow and black. The decrease in the usage of the black and yellow response categories resulting from usage of the brown response category can be seen in Figure 6. The black and yellow response functions from Session 1 (brown unavailable) are replotted alongside the black and yellow response functions from Session 4 (brown available). This figure demonstrates that, for 2 of the 3 subjects (B.L. and F.F.), the percentage of color allotted to the black response

\section{Percent}
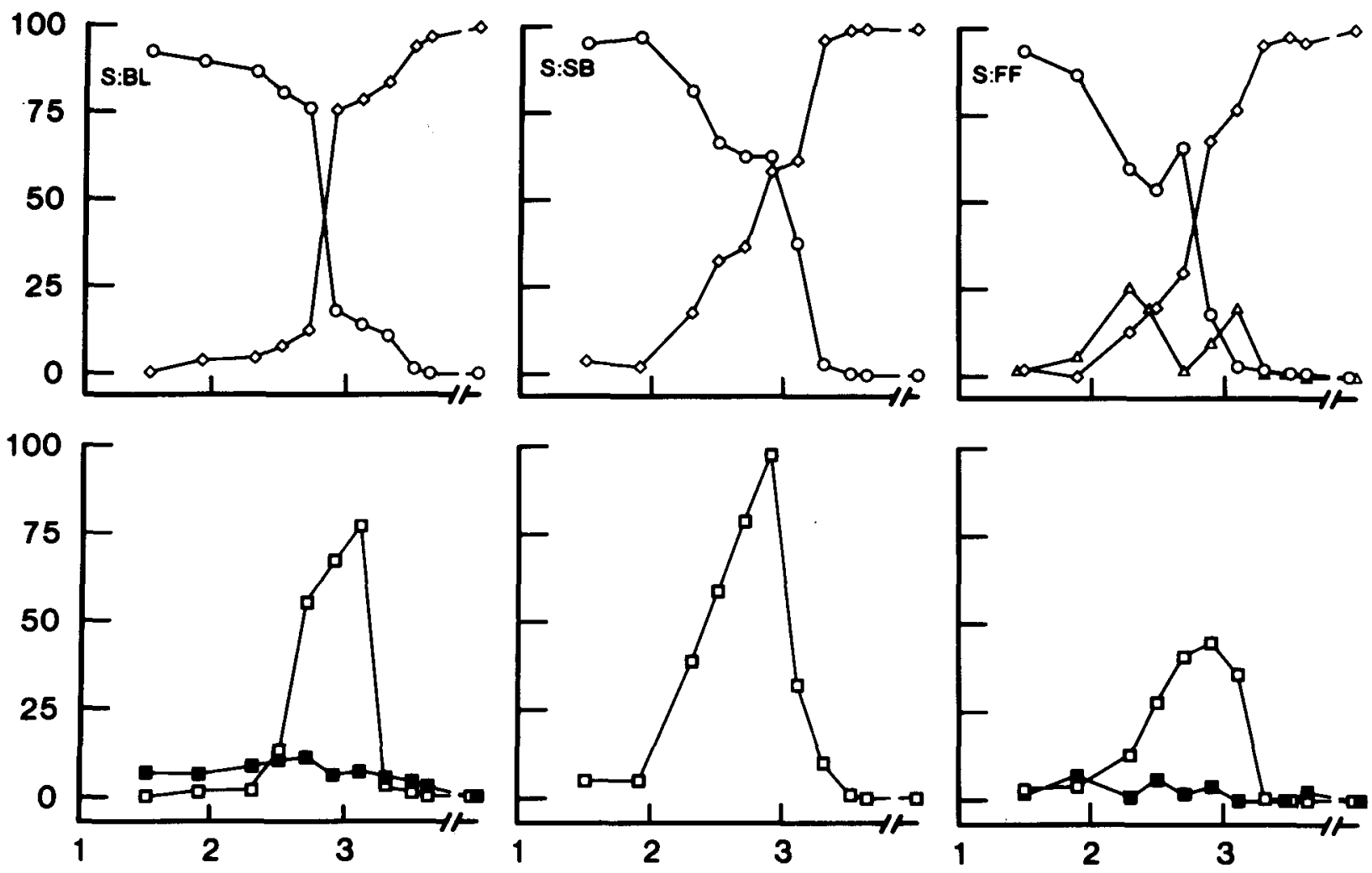

Surround Illuminance, Log TD

Figure 2. (Top panel) Color-naming responses from Session 1 of Experiment 2 with black, yellow, and red response functions indicated by diamonds, circles, and triangles, respectively. (Bottom panel) Computed functions from Session 1 and brown response functions from Session 4, with computed and brown response functions indicated by filled and unfilled squares, respectively. 


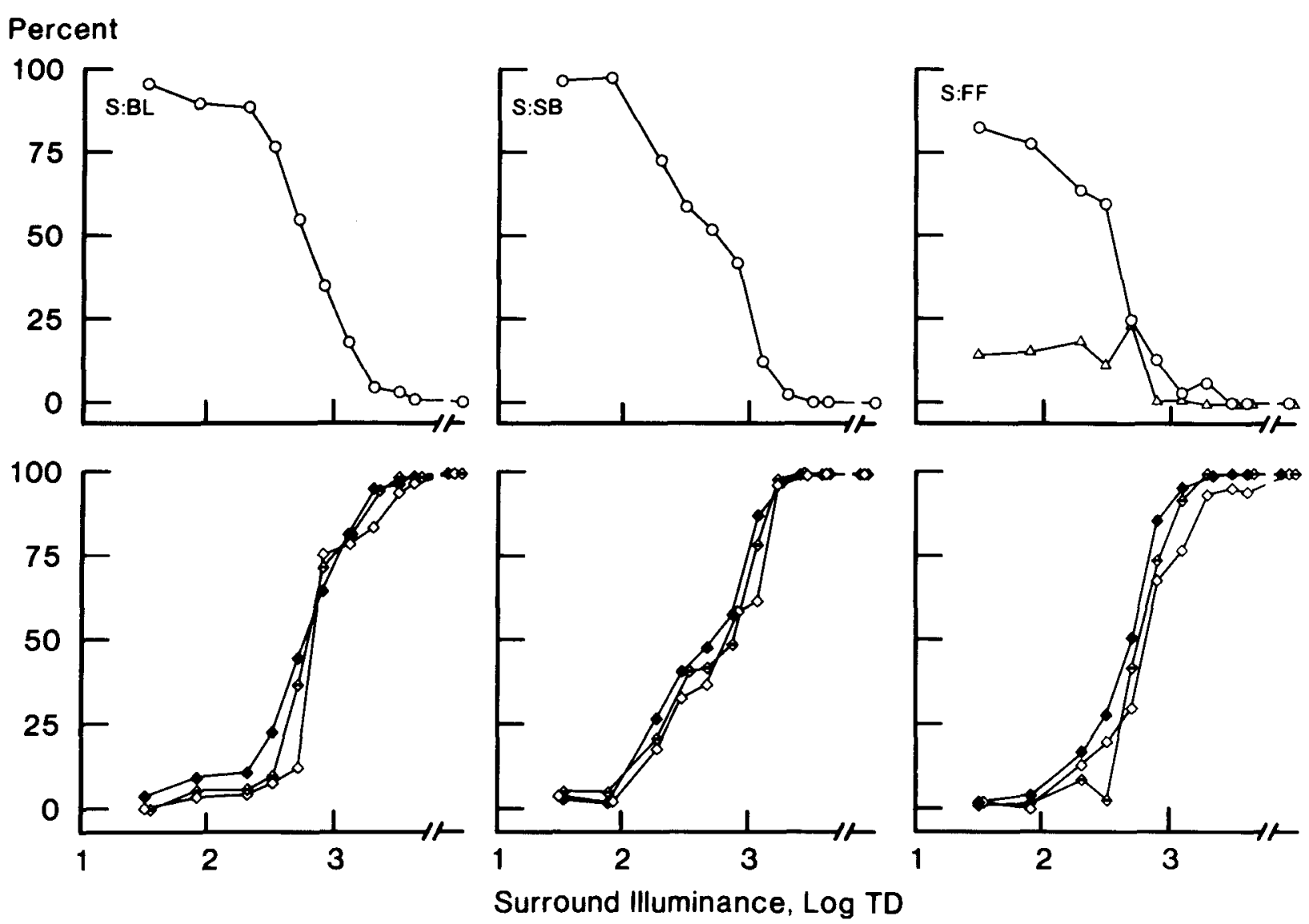

Figure 3. (Top panel) Color-naming responses from Session 2 of Experiment 2, with yellow and red response functions indicated by circles and triangles, respectively. (Bottom panel) Computed functions from Session 2 and black response functions from Sessions 1 and 3 , with computed functions represented by filled diamonds, and black response functions from Sessions 1 and 3 indicated by unfilled and cross-hatched diamonds, respectively.

category is reduced by the introduction of the brown response category, whereas the yellow response category is relatively unaffected. For the third observer (S.B.), the percentage of color allotted to both the yellow and black response categories is reduced by the introduction of the brown response category.

An interesting question prompted by the Fuld et al. (1983) results and the performance of Subject S.J.T. in Experiment 1 is whether subjects would be less willing to completely describe the series of stimuli with the terms yellow and black after they had used the term brown in Session 4. To answer this question, a fifth experimental session was conducted that was identical to the first session: the available response categories included black, yellow, and red. The results of this session are plotted simultaneously with those of Session 1 in Figure 7. It can be seen that the black and yellow (and red for Subject F.F.) response functions for the two sessions are in close agreement. Thus, even after the subjects had used the brown response category, they were still capable of completely describing the stimuli with the terms black and yellow. This provides even more evidence supporting the argument that brown is not an elemental color.

\section{GENERAL DISCUSSION}

The two experiments reported here were designed to (1) evaluate the degree to which continuous judgmental color-naming results are influenced by experimental design, especially by the order in which color response categories become available, and (2) investigate the possible elemental nature of the color brown. The maroon series of Experiment 1 was administered in an attempt to build in a dependency on the term maroon by allowing the subjects to use the term for several sessions and then depriving them of it. In Experiment 2, the term brown was never mentioned to the subjects until the second-tolast session. This was done to determine whether the subjects would require the term brown to describe the stimuli in earlier sessions (i.e., showing a computed function) before ever having used the term in the experiment.

The maroon-series results indicate that the order in which categories become available to subjects can directly influence color-naming results, and can build in a category dependence in some subjects. Subject S.J.T. showed a large, sharply peaking computed function when deprived of the term maroon; furthermore, this computed function 


\section{Percent}
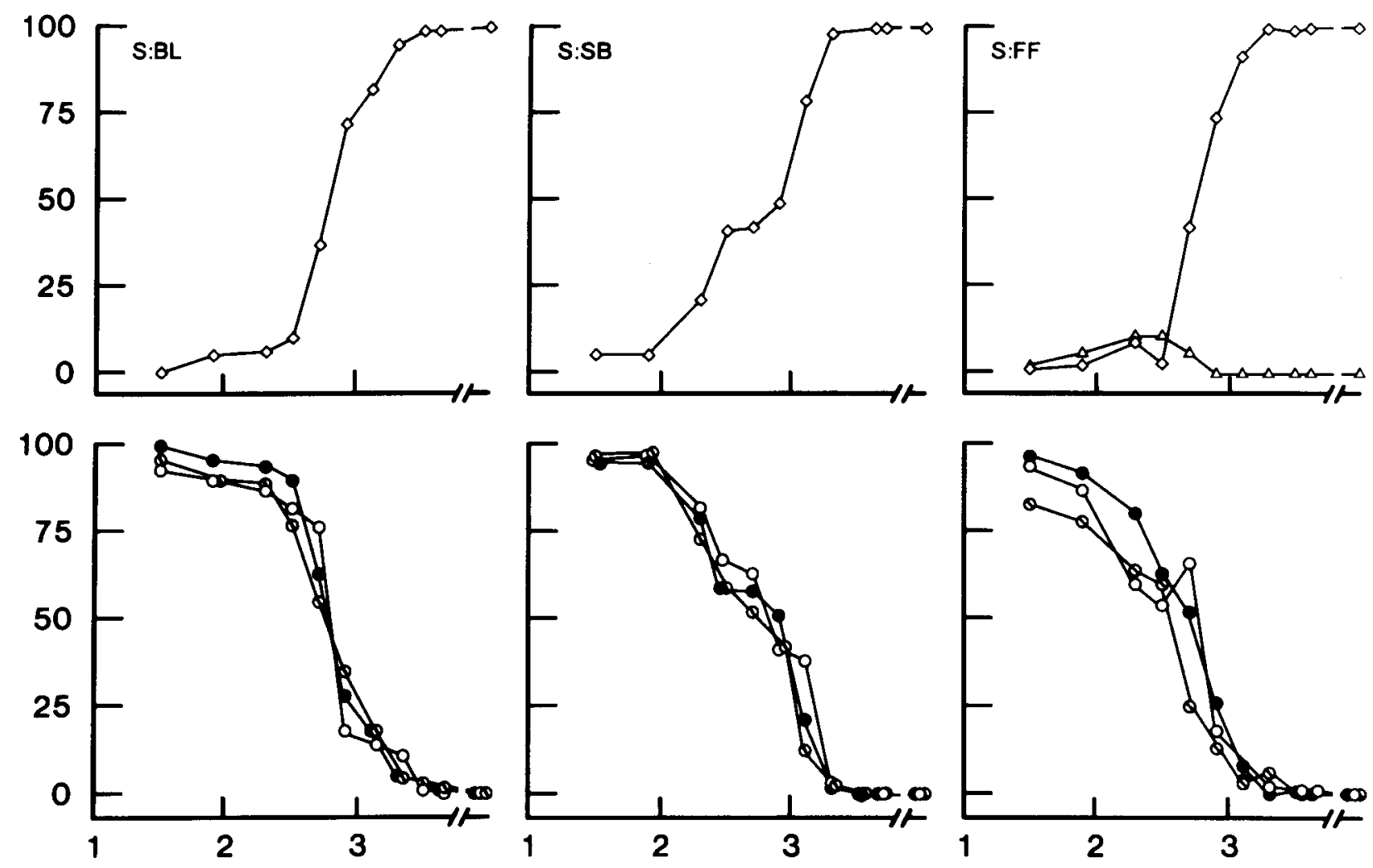

Surround Illuminance, Log TD

Figure 4. (Top panel) Color-naming responses from Session 3 of Experiment 2, with black and red response functions indicated by diamonds and triangles, respectively. (Bottom panel) Computed functions from Session 3 and yellow response functions obtained in Sessions 1 and 2, with computed functions represented by the filled circles, and yellow response functions from Sessions 1 and 3 indicated by unfilled and cross-hatched circles, respectively.

\section{Percent}
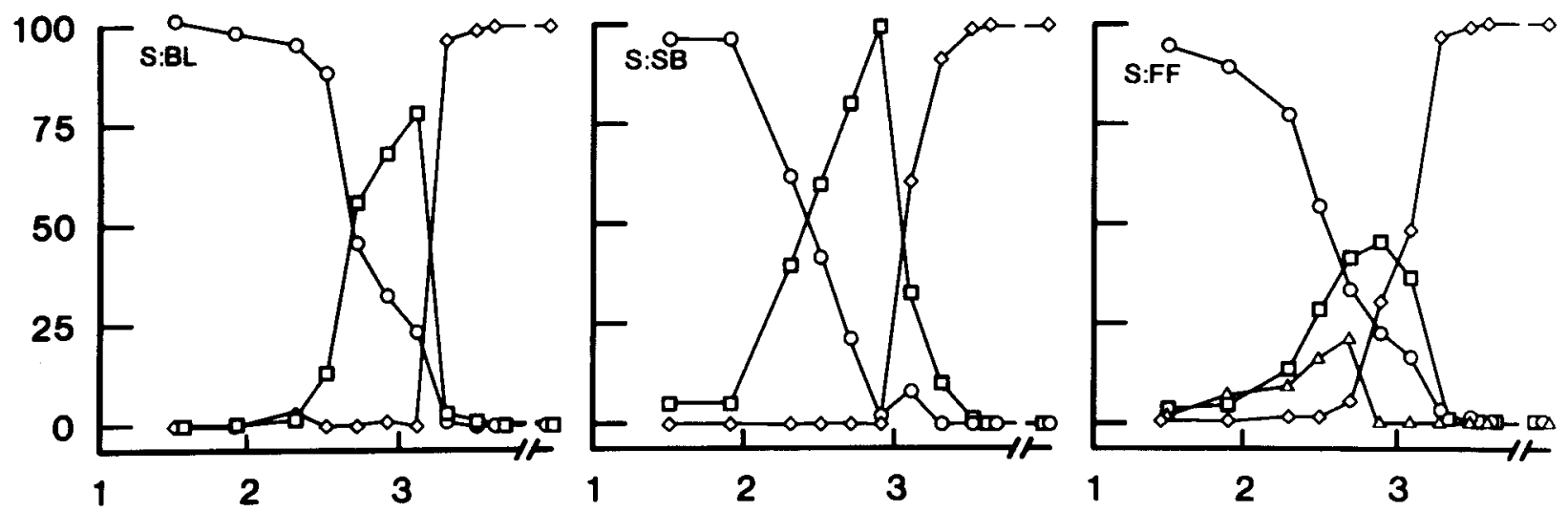

Figure 5. Color-naming responses from Session 4 of Experiment 2 with black, yellow, red, and brown response functions indicated by diamonds, circles, triangles, and squares, respectively. 


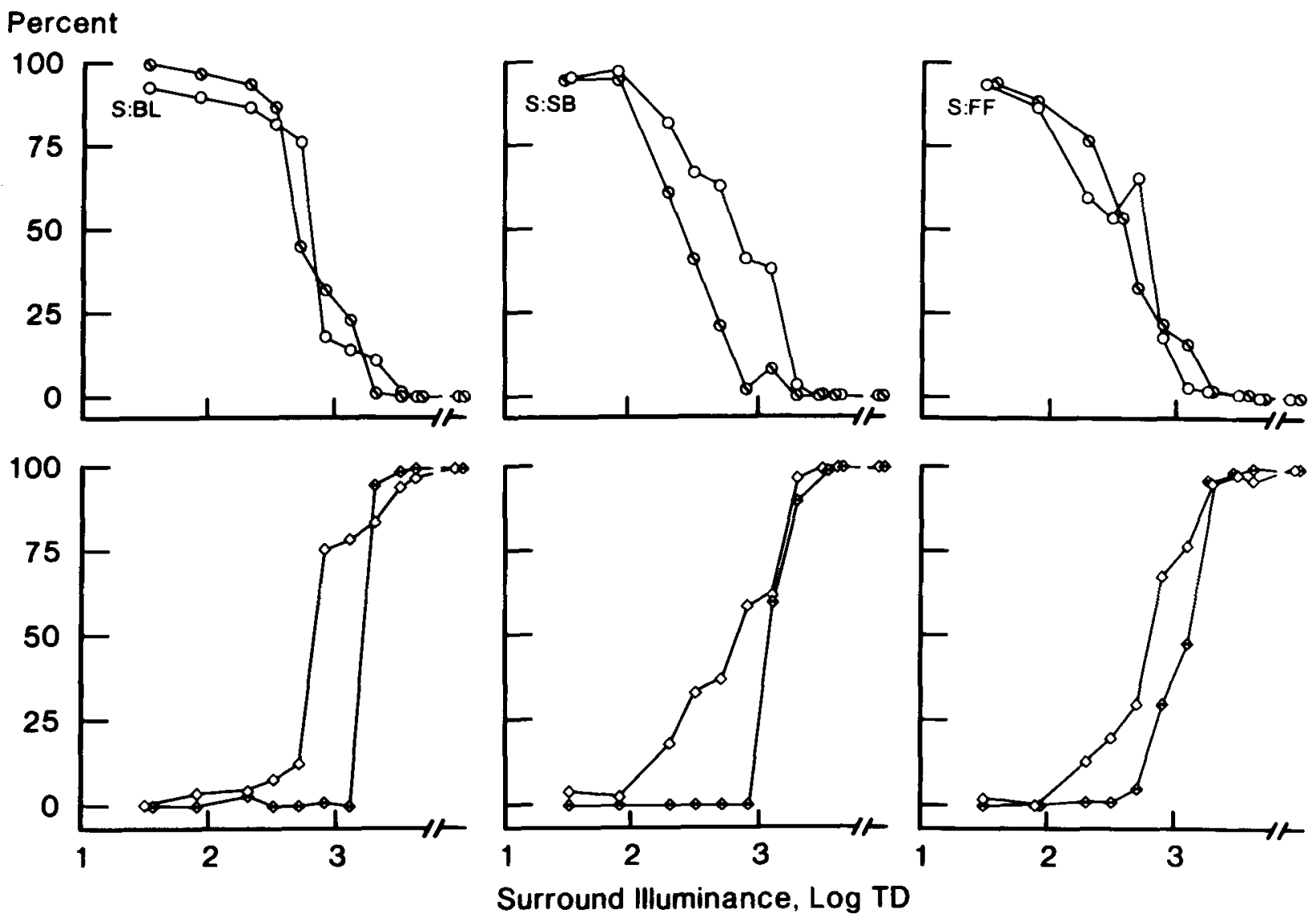

Figure 6. Comparison of yellow response functions (top panel) and black response functions (bottom panel) from Session 1, in which brown was not allowed (unfilled symbols), and from Session 4, in which brown was allowed (cross-hatched symbols). Yellow and black response functions are indicated by circles and diamonds, respectively.

\section{Percent}
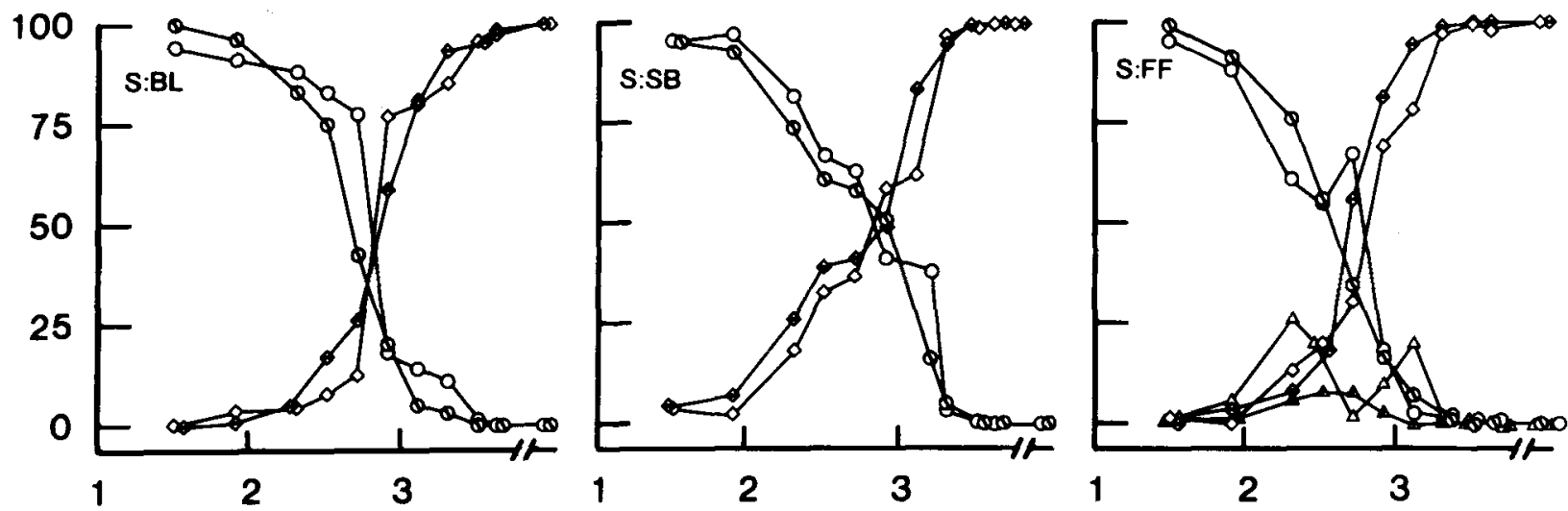

Figure 7. Comparison of yellow, black, and red response functions from the 2 sessions in which only these color terms were allowed (Sessions 1 and 5). Responses from Sessions 1 and 5 are indicated by the unfilled and cross-hatched symbols, respectively. Yellow, black, and red response functions are indicated by circles, diamonds, and triangles, respectively. 
closely matched his maroon response function. Fuld et al. (1983) had previously demonstrated that a similar set of stimuli could be fully described with the terms red and black, but Subject S.J.T., after using the term maroon in Sessions 1 and 2, was not willing to describe the stimuli in this manner.

In Experiment 2, brown failed to meet the criterion that establishes a color as elemental. Figure 1 clearly demonstrates this, as there was essentially no computed function present when both yellow and black were available. Yellow and black, on the other hand, were reconfirmed as elemental. Figures 2 and 3 show that when either of these terms was prohibited from use, computed functions were obtained that closely matched the yellow and black response functions obtained when the terms were available. Yellow and black (and red for Subject F.F.) were thus necessary and sufficient for describing the stimuli.

The data from the brown series do not substantiate the elemental nature of brown that was suggested by Fuld et al. (1983). Their subjects may have been operating with a response bias of the sort displayed by Subject S.J.T. in Experiment 1. Allowing the subjects to use the color name brown in the preliminary and first sessions appears to have made them reluctant to give it up in later sessions. The contrast between the present brown series results and those of Fuld et al. thus corroborates the conclusions drawn from the maroon series results: The order in which color categories become available can have a significant effect on the results, at least for some subjects.

Writers and theorists have speculated about whether brown is elemental or not, but the paper by Fuld et al. (1983) and our study seem to be the only empirical attempts to resolve the issue. Unfortunately, neither attempt is definitive. The results of Fuld et al. are consistent in terms of brown's being elemental or nonelemental, but exceedingly difficult to resolve into components. Our data are consistent with brown's being a perceptual combination of yellow and black. It is possible, however, to assert that by having the subjects use yellow and black before they saw brown, we trained them to artificially fractionate the elemental color brown. While there is no airtight logical or empirical defense against this possibility, it is fair to cite Sternheim and Boynton's (1966) original study, in which no elemental category was fractionated even when preceded in the stimulus series by adjacent hues. In fact, we know of no demonstration that subjects can be trained to break down an elemental color in a way that accurately simulates how nonelemental colors are described. In view of this, and considering the success of the opponent-process model in describing all other areas of color space, we conclude that it is best to tentatively regard brown as a perceptual combination of the elemental colors yellow and black.

\section{REFERENCES}

Bartleson, C. J. (1976). Brown. Color Research \& Application, 1, 181-191.

BorIng, E. G. (1942). Sensation and perception in the history of experimental psychology. New York: Appleton-Century-Crofts.

Committee on Colorimetry of the Optical Society of America. (1963). The science of color. New York: Thomas Y. Crowell.

Fuld, K., Werner, J. S., \& Wooten, B. R. (1983). The possible elemental nature of brown. Vision Research, 23, 631-637.

Fuld, K., Wooten, B. R., \& Whalen, J. J. (1981). Elemental hues of short-wave and extraspectral lights. Perception \& Psychophysics, 29, 317-322.

HERING, E. (1964). Outlines of a theory of light sense (L. M. Hurvich \& D. Jameson, Trans.). Cambridge, MA: Harvard University Press. (Original work published 1920)

Hurvich, L. M. (1981). Color vision. Sunderland, MA: Sinauer Associates.

KNOBLAUCH, K. (1981). Analysis of opponent interactions in sex-linked dichromacy. Unpublished doctoral dissertation, Brown University, Providence, RI.

Padgham, C. A., \& Saunders, J. E. (1975). The perception of light and colour. New York: Academic Press.

QuinN, P. C., Wooten, B. R., \& Ludman, E. J. (1985). Achromatic color categories. Perception \& Psychophysics, 37, 198-204.

RATLIF, F. (1976). On the psychophysiological bases of universal color terms. American Philosophical Society, 120, 311-330.

STERNHEIM, C. E., \& BOYNTON, R. M. (1966). Uniqueness of perceived hues investigated with a continuous judgmental technique. Journal of Experimental Psychology, 72, 770-776.

Webster's Third New International Dictionary of the English Language. (1961). Springfield, MA: G. \& C. Merriam.

Westheimer, G. (1966). The Maxwellian view. Vision Research, 6, 669-682.

\section{NOTE}

1. For Figures 1 through 7, response functions with peaks of $3 \%$ or less are not plotted.

(Manuscript received October 13, 1986; revision accepted for publication August 4, 1987.) 